\title{
Flow Boiling Characteristics in a Microchannel with Various Wettability- Patterned Surfaces
}

\author{
Hongzhao Wang, Yinchuang Yang, Huihe Qiu \\ Department of Mechanical and Aerospace Engineering \\ The Hong Kong University of Science and Technology \\ Clear Water Bay, Kowloon, Hong Kong \\ meqiu@ust.hk
}

\begin{abstract}
Flow boiling in a microchannel is an effective and attractive solution for thermal management. In this work, flow boiling was performed in microchannels with various wettability-patterned dotted surfaces. Pitch distances of dots were changed to study the wettability pattern effects on microchannel flow boiling. Glass-silicon-glass microchannels integrated with internal platinum heaters and various wettability patterns were fabricated and characterized using MEMS techniques. The transparent top of the channel allowed visualization of bubble dynamics and flow patterns. The hydraulic diameter of the microchannel is $338 \mu \mathrm{m}$, the side length of hydrophobic dots is $72 \mu \mathrm{m}$ and the pitch distances of hydrophobic dots change from $122 \mu \mathrm{m}$ to $172 \mu \mathrm{m}$. Bubble dynamics and flow patterns were visualized using a high-speed camera and a microscope. Heat transfer performance, including boiling curves and heat transfer coefficients $(H T C)$, were explored experimentally under different mass flux. It is found that bubbles coalesce more frequently and flow pattern transitions are more intense with the decrease of pitch distance. In the low mass flux region, better heat transfer capability can be observed on the surface with larger pattern pitch distance, while with the increase of mass flux, a microchannel with smaller dot pitch distance presents better heat dissipation ability. Moreover, the critical heat flux $(\mathrm{CHF})$ decreases with the decrease of dot pitch distance. The reasons behind these phenomena are related to the bubble coalescence, bubble density, and flow movement.
\end{abstract}

Keywords: Flow Boiling, Pitch Distance, Wettability-pattern, Heat Transfer

\section{Introduction}

Flow boiling in microchannels is a promising cooling technique in the electronics industry as it can obtain good heat transfer performance without large surface temperature oscillation [1][2]. The majority of researchers have studied homogeneous wettability microchannels. However, there are dilemmas about homogeneous wettability microchannels. On the one hand, a hydrophobic surface reduces bubble departure frequency, which decreases $C H F$. On the other hand, a hydrophilic surface decreases bubble nucleate sites [3], which diminishes the HTC. In order to tackle these problems, a wettability-patterned surface, which contains hydrophobic dots and hydrophilic networks, has been proposed [4] to manipulate nucleation boiling in a micro fluid flow channel.

It is well known that the morphologies of the patterns affect the heat transfer performance in pool boiling [5][6]. The dot size and pitch distance have a dominant effect at low heat flux, while the number of dots is more important at high heat flux [6]. However, the relationship between pattern morphologies and flow boiling heat transfer remains unknown. In this paper, the effect of pitch distance on microchannel flow boiling heat transfer will be studied. Bubble dynamics and flow patterns will be captured and the heat transfer performance will be explored experimentally.

\section{Experimental Setup}

Fig. 1 shows the experimental setup. It consists of four parts: a fluid loop, a test part, a data collection part and a visualization part. The working fluid, deionized water, which had already been degassed in a vacuum chamber for more than $12 \mathrm{~h}$, was pushed into the microchannel by a syringe pump (Model 33, Harvard Apparatus Co.). A standard resistor (MP2000, $2 \mathrm{ohm}$ ), which connected in series with the heater, was measured to gain the voltage across the resistor. Two Ttype thermocouples were used to measure the inlet/outlet water temperature. The voltage of the heater $V_{h}$, the voltage of the standard resistor $V_{r}$ and the inlet/outlet temperature were collected by a data acquisition switch unit (Agilent 34970A). A microscope (Nikon eclipse Ni-E) connected with a high-speed camera (FASTCAM SA-Z) was employed to visualize the 
bubble dynamics during flow boiling. Before the experiment, the heater was calibrated in an oven to obtain the relationship between the resistance and the temperature. The heat loss was measured using Chen and Chung's method [7]. The mass flux $G$, the heat flux $q_{\text {eff }}$ and the $H T C$ were computed using equations (1) to (3), where $M, \rho$ and $A_{\text {cro }}$ are the volumetric flow rate, the fluid density, and the cross-section area of the microchannels, respectively. $R$ is the resistance of the standard resistor. $A_{h}$ is the surface area of the heater. $T_{h}$ is the heater wall average temperature and $T_{\text {sat }}$ is the saturation temperature.

$$
\begin{gathered}
G=\frac{M \rho}{A_{\text {cro }}} \\
q_{\text {eff }}=q_{\text {total }}-q_{\text {loss }}=\frac{V_{h} /_{R}-Q_{\text {loss }}}{A_{h}} \\
H T C=\frac{q_{\text {eff }}}{T_{h}-T_{\text {sat }}}
\end{gathered}
$$

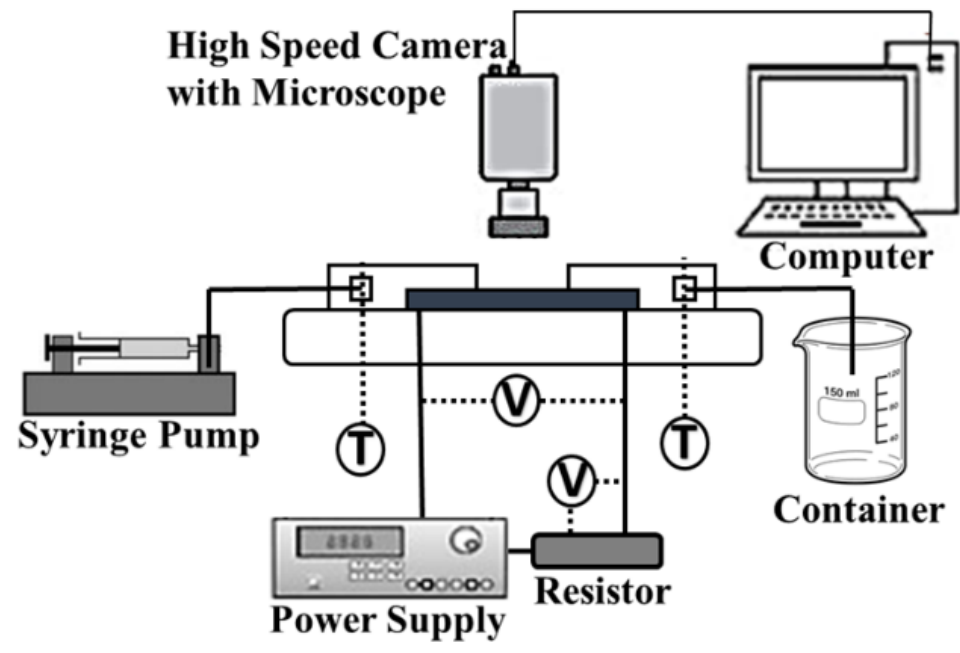

Fig. 1: Schematic of experimental setup.

\section{Results and Discussion}

\subsection{Bubble Dynamics Visualization}

The hydrophobic square dots on three different surfaces have the side length of $72 \mu \mathrm{m}$ and the pitch distances of 122 , 144 and $172 \mu \mathrm{m}$, respectively. These three types of surfaces are named P122, P144 and P172, respectively. A series of high speed images of flow patterns on different surfaces are shown in Fig. 2. It is clear that bubbles prefer to nucleate on the hydrophobic dots. In addition, flow pattern transition on P122 surfaces are faster than the other surfaces. This is due to the more rapid coalescence of the bubbles on the surface with smaller pitch distances, which causes the stronger temperature fluctuation. 


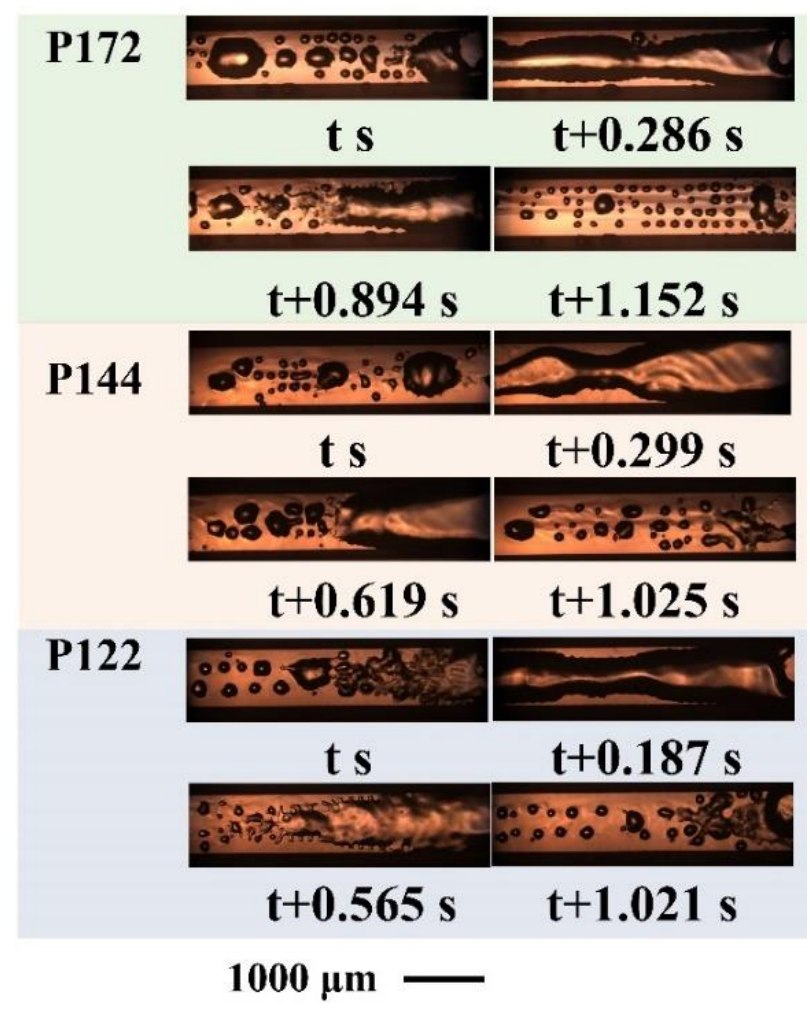

Fig. 2: High speed flow patterns images of microchannels with various wettability patterns under the conditions of $G=164.4 \mathrm{~kg} / \mathrm{m}^{2} \mathrm{~s}$ and $q_{\text {eff }}=101.2 \mathrm{~W} / \mathrm{cm}^{2}$ for P122, $q_{\text {eff }}=102.1 \mathrm{~W} / \mathrm{cm}^{2}$ for P144 and $q_{\text {eff }}=101.6 \mathrm{~W} / \mathrm{cm}^{2}$ for P172.

\subsection{Heat Transfer Study}

Fig. 3 shows the boiling curves and $H T C$ curves for three surfaces at different mass flux. It can be seen that at low mass flux (when $G=41.1 \mathrm{~kg} / \mathrm{m}^{2} \mathrm{~s}$ ), P172 presents best heat transfer performance, while P122 shows the worst heat removal capability. This is because at low mass flux, bubbles on the P122 surface are easily merged before they depart, which lead to larger dryout spot and worse performance. However, at the higher mass flux (when $G=246.6 \mathrm{~kg} / \mathrm{m}^{2} \mathrm{~s}$ ), the heat dissipation ability of P122 microchannel outweighs the rest. At the higher mass flux, more bubbles depart before they merge and as more bubbles are nucleated from the P122 microchannel (because it has the most nucleation sites), therefore, P122 microchannels have the best performance in the nucleated flow boiling regimes. In addition, the HTCs show decreasing trends, which are consistent with previous reports [8][9][10]. Moreover, CHFs of microchannel P172 are higher at both mass flux. Equation (4) shows the surface tension force calculation [11][12], where $F_{s}, \sigma, L, A_{\text {cro }}, \varnothing, \theta_{\text {ro }}$ and $\theta_{t f}$ are surface tension force, surface tension, relevant bubble dimension, channel cross-sectional area, area fraction of hydrophilic area, equilibrium contact angle of silicon oxide and Teflon, respectively. The calculation result shows that P172 has the largest surface tension force, which means that it has better rewetting capability of the surface. Thus, it is favourable to the $C H F$ s.

$$
F_{S}=\frac{\sigma\left[\cos \theta_{r o} \emptyset+\cos \theta_{t f}(1-\emptyset)\right] L}{A_{\text {cro }}}
$$



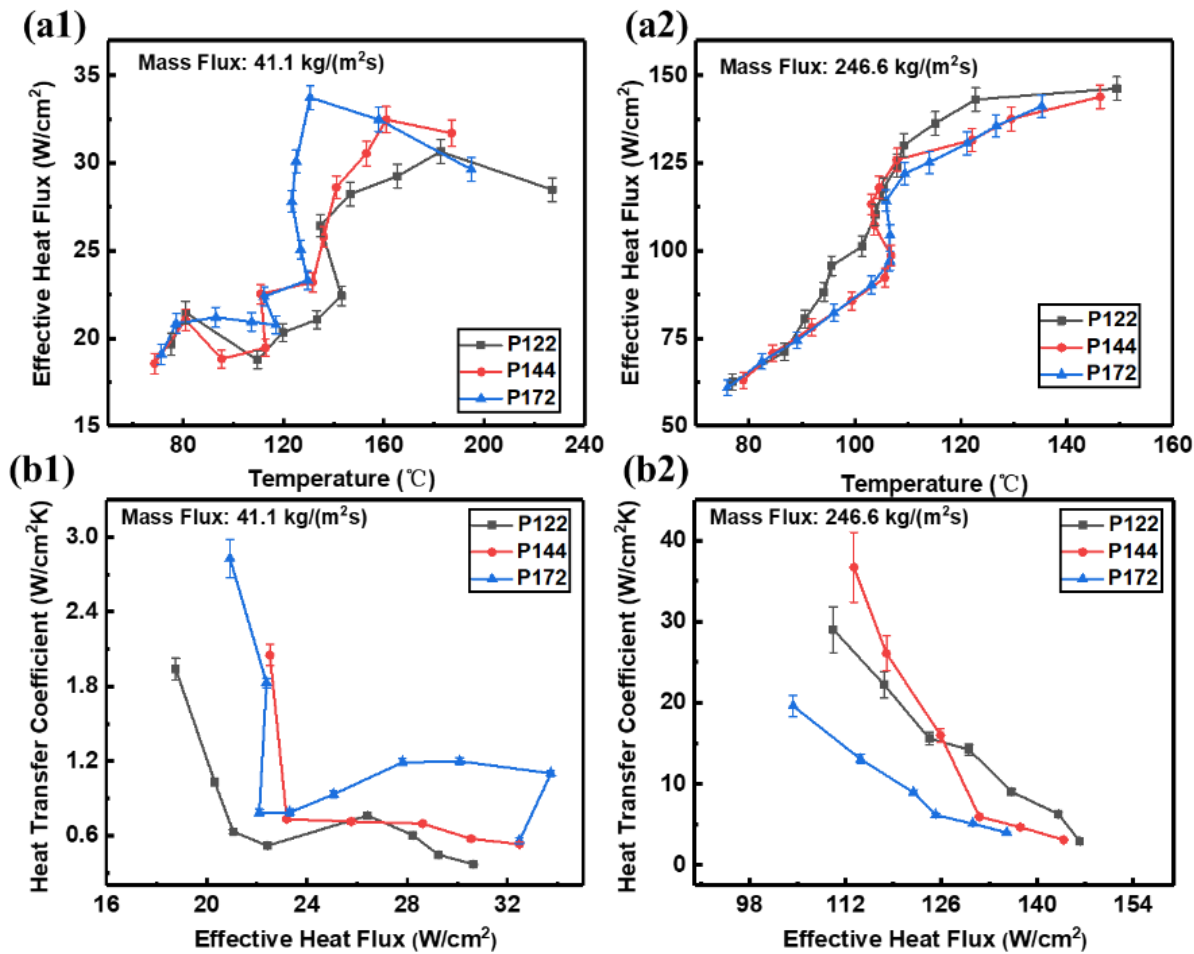

Fig. 3: (a1) Boiling curve at $G=41.1 \mathrm{~kg} / \mathrm{m}^{2} \mathrm{~s}$; (a2) boiling curve at $G=246.6 \mathrm{~kg} / \mathrm{m}^{2} \mathrm{~s}$; (b1) $H T C$ versus $q_{\text {eff }}$ at $G=41.1 \mathrm{~kg} / \mathrm{m}^{2} \mathrm{~s}$; (b2) $H T C$ versus $q_{e f f}$ at $G=246.6 \mathrm{~kg} / \mathrm{m}^{2}$ s for P122, P144 and P172 microchannels.

\section{Conclusion}

In this paper, bubble dynamics and heat transfer of microchannels with wettability-patterns of various pitch distances were explored and discussed. It is found that bubbles coalesce more easily and flow patterns change faster in the microchannel with smaller dot pitch distance. With the increase of mass flux, microchannel with smaller dot pitch distance have a better heat transfer performance compared to the microchannel with larger dot pitch distance.

\section{Acknowledgements}

This work was supported by the Research Grants Council of the Government of Hong Kong Special Administrative Region (HKSAR) with RGC/GRF Project No. 16205018. We acknowledge the assistance from the Nanosystem Fabrication Facility (NFF) of HKUST for the device / system fabrication.

\section{References}

[1] T. G. Karayiannis and M. M. Mahmoud, "Flow boiling in microchannels: Fundamentals and applications," Appl. Therm. Eng., vol. 115, pp. 1372-1397, Mar. 2017.

[2] D. Deng, W. Wan, Y. Qin, J. Zhang, and X. Chu, "Flow boiling enhancement of structured microchannels with micro pin fins," Int. J. Heat Mass Transf., vol. 105, pp. 338-349, Feb. 2017.

[3] C. S. Sujith Kumar, S. Suresh, L. Yang, Q. Yang, and S. Aravind, "Flow boiling heat transfer enhancement using carbon nanotube coatings," Appl. Therm. Eng., vol. 65, no. 1-2, pp. 166-175, Apr. 2014.

[4] H. Wang, Y. Yang, M. He, and H. Qiu, "Subcooled flow boiling heat transfer in a microchannel with chemically patterned surfaces,” Int. J. Heat Mass Transf., vol. 140, pp. 587-597, Sep. 2019.

[5] H. Jo, S. Kim, H. Kim, J. Kim, and M. H. Kim, "Nucleate boiling performance on nano/microstructures with different wetting surfaces," Nanoscale Res. Lett., 2012. 
[6] H. Jo, H. S. Ahn, S. Kang, and M. H. Kim, "A study of nucleate boiling heat transfer on hydrophilic, hydrophobic and heterogeneous wetting surfaces," Int. J. Heat Mass Transf., vol. 54, no. 25-26, pp. 5643-5652, Dec. 2011.

[7] T. Chen and J. N. Chung, "An experimental study of miniature-scale pool boiling," J. Heat Transfer, 2003.

[8] D. Li, G. S. Wu, W. Wang, Y. D. Wang, D. Liu, D. C. Zhang, Y. F. Chen, G. P. Peterson, R. Yang, "Enhancing Flow Boiling Heat Transfer in Microchannels for Thermal Management with Monolithically-Integrated Silicon Nanowires," Nano Lett., vol. 12, no. 7, pp. 3385-3390, Jun. 2012.

[9] D. Deng, W. Wan, H. Shao, Y. Tang, J. Feng, and J. Zeng, "Effects of operation parameters on flow boiling characteristics of heat sink cooling systems with reentrant porous microchannels," Energy Convers. Manag., vol. 96, pp. 340-351, May 2015.

[10] Z. Wu and B. Sundén, "On further enhancement of single-phase and flow boiling heat transfer in micro/minichannels," Renew. Sustain. Energy Rev., vol. 40, pp. 11-27, Dec. 2014.

[11] T. Alam, W. Li, F. Yang, W. Chang, J. Li, Z. Wang, J. Khan, C. Li, "Force analysis and bubble dynamics during flow boiling in silicon nanowire microchannels," Int. J. Heat Mass Transf., vol. 101, pp. 915-926, Oct. 2016.

[12] A. B. D. Cassie and S. Baxter, "Wettability of porous surfaces," Trans. Faraday Soc., 1944. 